\title{
HUMOR ALEJANDRINO EN EL MIMIAMBO 5 DE HERODAS
}

This paper reveals the humoristic content of two passages of Mimiamb $\mathbf{V}$ by Herodas (vv. 39 and 17).

Uno de los recursos característicos del que a menudo se sirven los poetas alejandrinos es el de introducir en sus composiciones efectos humorísticos ${ }^{1}$. Dentro de este grupo de poetas también podemos incluir la figura de Herodas (Hds.) ${ }^{2}$. No es por ello nada raro que en sus $\mathrm{Mi}$ miambos encontremos algunos testimonios de esta práctica. Sin embargo y a pesar de las sucesivas ediciones y comentarios, no siempre se ha sabido reconocer la intención humorística del poeta en determinados pasajes. Ello es debido en buena medida a la particular naturaleza de este humor, fruto del carácter docto y erudito de quien lo provoca y que hace que no sea siempre fácil reconocerlo. En efecto, estos poetas recurren a sutiles alusiones o a significados más raros de algunos vocablos que, por esa misma razón, pasan a veces inadvertidos al lector no atento. La certera advertencia de Headlam, «there is more in Herodas than meets the unpractised eye», puede aplicarse al resto de los eruditos poetas alejandrinos ${ }^{3}$. Con estas premisas, nos proponemos en este ar-

' Sobre este tema, cf., entre otros trabajos de G. Giangrande, L'humour des Alexandrins, Amsterdam 1975, y "Polisemia del linguaggio nella poesia alessandrina", QUCC 24, 1977, pp. 97-106, en los que se estudia y ejemplifica la particular naturaleza del humor de estos poetas eruditos.

2 Cf. W. Headlam-A. D. Knox, Herodas. The Mimes and Fragments, Cambridge 1922, p. XXVII, donde se considera ya a Hds. como "a true Alexandrian». A pesar de ello, y debido quizá a una visión superficial y deformada de sus Mimiambos, considerados simplemente como obras menores que tratan temas poco decentes, la opinión que se ha formado en general sobre Hds. no suele ser muy positiva. Como muestra, A. Lesky, en su difundida Historia de la literatura griega, Madrid 1976, p. 778 , duda incluso si concederle el simple nombre de poeta. Por nuestra parte creemos haber ratificado claramente para Hds. su condición de poeta doctus en nuestra tesis doctoral, Estudio del léxico de Herodas y sus fuentes (Universidad de Oviedo 1991, inédita).

${ }^{3}$ Cf. Headlam-Knox, p. X. 
tículo desvelar en dos pasajes del mimiambo $\mathrm{V}$ de Hds. la carga humoristica que comportan y que, desde nuestro punto de vista, no se ha sabido apreciar completamente hasta ahora.

El primero de los dos pasajes que analizaremos es el del verso 39. En los versos anteriores vemos al esclavo Gastrón tratando de justificarse ante su dueña Bitina, con la que mantiene relaciones amorosas, de la acusación que ésta le hace de que anda coqueteando también con otra mujer, una tal Anfitea, y que es lo que provoca en ella el ataque de celos que Hds. nos pinta a lo largo del mimiambo. En medio de sus excusas, Gastrón es sorprendido por Bitina en una abierta contradicción (cf. vv. 26-27 y 35-38) y es entonces cuando el esclavo justifica su actitud diciendo que lo que pretendía era aplacar la ira de su ama: v.

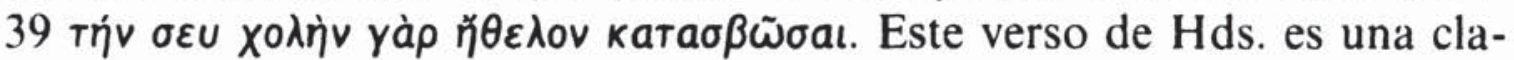
ra reminiscencia homérica. El poeta, mediante una ligera uariatio ${ }^{4}$, hace

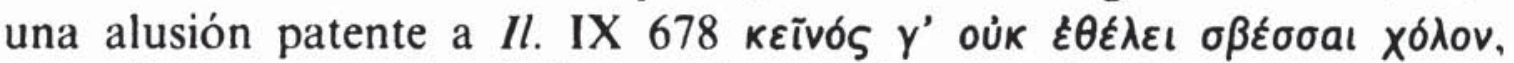
mensaje que transmite Odiseo a Agamenón, en relación con Aquiles y la negativa de éste a deponer su cólera. Es fácil percibir la equivalencia

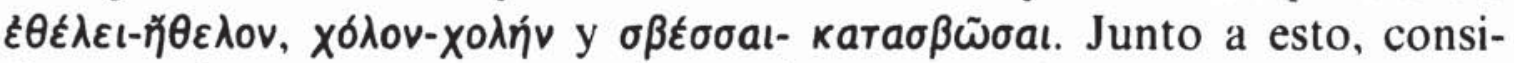

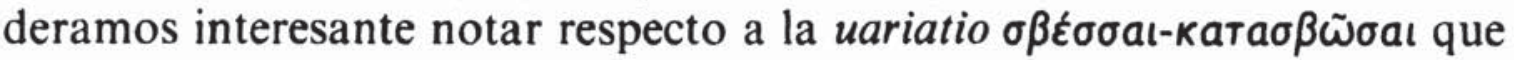

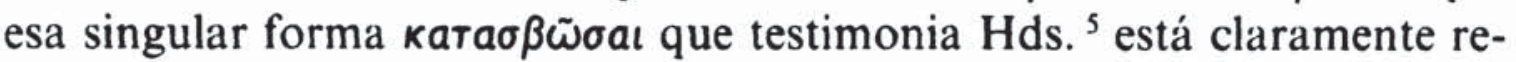

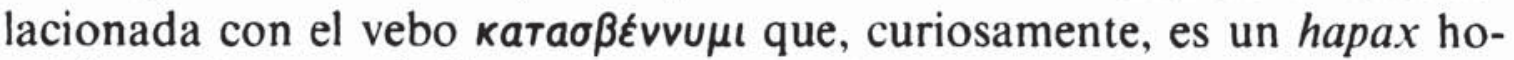
mérico ${ }^{6}$. Que nos hallamos en este v. 39 ante una alusión homérica es

${ }^{4}$ Como repetidas veces ha hecho ver el Prof. G. Giangrande, el empleo de la imitatio cum uariatione respecto a Homero es uno de los más típicos recursos de la poesía alejandrina: "gli alessandrini, come canone basilare della loro arte allusiva, preferiscono non riprodurre ciò che in Omero è normale ed usuale, bensi o riprodurre ciò che in Omero è eccezionale o unico, oppure applicare i canoni di imitatio cum uariatione ed oppositio in imitando...", cf. G. Giangrande, "Problemi testuali nei poeti Alessandrini", La critica testuale greco-latina oggi. Metodi e problemi. Atti del Convegno Internazionale, Napoli 29-31 ottobre 1979, Roma 1981, p. 383.

5 Sobre esta forma verbal, cf. I. C. Cunningham, Herodas. Mimiambi, Oxford 1971, p. 153, y V. Schmidt, Sprachliche Untersuchungen zu Herondas, Berlín 1968, pp. 84-88. En el index uerborum de su citada edición oxoniense, p. 233, Cunningham lematiza la palabra como катабßóa, mientras que en su más reciente edición, Hero-

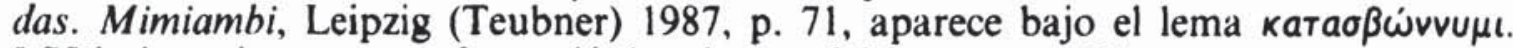

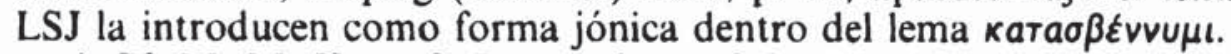

${ }^{6}$ Cf. M. M. Kumpf, Four Indices of the Homeric Hapax Legomena, Hildesheim 1984, p. 66, donde menciona a Hds. Este empleo de hapax y otras rarezas homéricas es asimismo una de las características más frecuentes entre los poetae docti alejandrinos sobre las que ha insistido una vez más el Prof. Giangrande, cf. "Hellenistic Poetry and Homer», $A C$ 39, 1970, p. 65. Véase asimismo, a propósito del idilio XXV de Teócrito, la constatación de G. Chrysaffis, A Textual and Stylistic Commentary on Theocritus' Idyll XXV, Amsterdam 1981, p. 12: "The poet's tendence to use Homeric hapax legomena, rarities and disputed words and expresions is obvious throughout." 
algo que ya había sido percibido por más de un comentarista ${ }^{7}$, pero no hemos visto, en cambio, que ninguno haya reparado en la posibilidad de que exista además un toque de humor. Sin embargo, nos parece claro que la alusión homérica no es gratuita sino que está hecha intencionadamente por Hds. con un fin humorístico. Como hemos dicho, el humor es algo presente en $\mathrm{Hds}$. al igual que en otros poetas alejandrinos ${ }^{8}$. Pues bien, la comicidad que encierra este v. 39 y que en nuestra opinión no ha sabido percibirse hasta ahora, es que las palabras pronunciadas por Gastrón, debido a sus evidentes conexiones homéricas, vienen a suponer la equiparación de la cólera de Bitina ni más ni menos que con la cólera de Aquiles, lo cual resulta evidentemente grotesco. Nos parece que estamos ante un ejemplo muy bueno de la sutileza artística de Hds. con la que es capaz de incluir en un contexto tan distinto al homérico una expresión que lo recuerda claramente, al tiempo que provoca un efecto cómico.

El segundo de los pasajes en los que creemos que se puede detectar otro efecto humorístico está unos pocos versos antes, en concreto en el

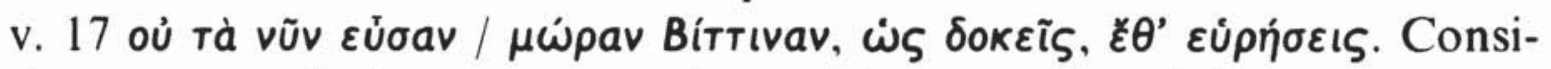
deramos, en efecto, que se puede postular para la palabra $\mu \omega \dot{\rho} \rho \mathbf{v}$ una doble alusión con intenciones humorísticas por parte de Hds. El empleo intencionado ${ }^{9}$ de esta palabra con fines humorísticos por Hds., que aquí pondremos de manifiesto, ha pasado inadvertido hasta ahora ya que tampoco lo hemos visto apreciado en ningún comentario de nuestro autor. Pues bien, dejando a un lado la cuestión del acento de la forma $\mu \omega ́$ pav ${ }^{10}$, nos interesa ahora centrarnos en el significado de la misma. Esta palabra alude principalmente a la estupidez o insensatez de una persona, pero también, en uno de sus usos menos frecuentes pero suficientemente constatado, puede referise a la lascivia. De esta manera, cuando Bitina pronuncia las palabras antes citadas del v. 17 en apariencia está diciendo simplemente que "no es una estúpida», pero el lector

' Cf. N. Terzaghi, Eroda. I Mimiambi, Turin 1925, p. 106; P. Groeneboom, Les Mimiambes d'Hérodas, Roma 1973 (reimp.), p. 165, «l'expression est déjà homérique»; G. Puccioni, Herodae Mimiambi, Florencia 1950, p. 102; Cunningham, p. 153.

${ }^{8}$ Como dice el Prof. Giangrande, "Herodas' humour has many features in common with Theocritus" y "The poet knows how to employ irony and puns", cf. "Interpretation of Herodas", QUCC 15, 1973, pp. $92-94$ (con sus nn. 32 y 34) y 98.

${ }^{9}$ Obsérvese a este respecto cómo en III 27 y en la misma posición métrica en principio del coliambo emplea en cambio Hds. el adj. ävouv para referirse asimismo a la insensatez, en ese caso de Metrotime.

10 Sobre la acentuación de esta palabra cf. Cunningham, p. 151, y V. Schmidt, pp. 18-19. No parece correcta $\mu \tilde{\omega} \rho a v$, que Terzaghi, p. 101, considera una "forma impossibile». 
erudito a quien iban dirigidos estos poemas " no puede dejar de percibir que esas mismas palabras encierran un doble significado ya que puede entenderse a su vez que Bitina está afirmando que "no es una lasciva». Y nos parece claro que esto último provoca un efecto cómico ya que choca frontalmente con su manera de ser, pues Hds. nos la presenta desde el principio como una mujer entregada a las relaciones sexuales

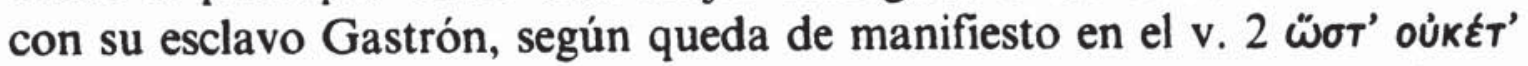

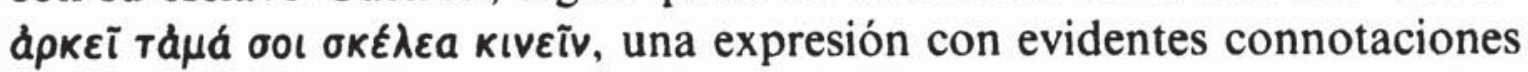
obscenas $^{12}$. Para ilustrar esta interpretación que proponemos para el v. 17 de Hds. son decisivos dos pasajes de Eurípides, a quien, sin duda, Hds. está siguiendo en cuanto a este juego alusivo con la palabra

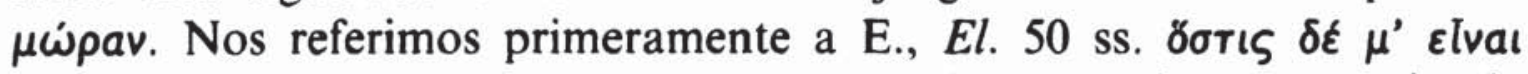

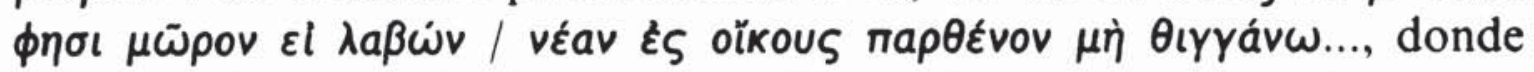
$\mu \tilde{\omega} \rho v$ encierra una doble alusión análoga a la que aquí descubrimos en Hds. En efecto, como acertadamente anota J. L. Calvo, esta expresión de Euripides es una "frase sólo inteligible si se tiene en cuenta que $\mu \tilde{\omega}$ pos significa 'bobalicón' pero también 'lascivo', etc. (en oposición a

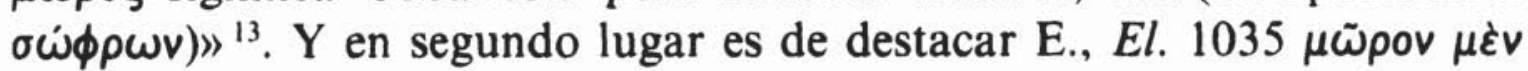

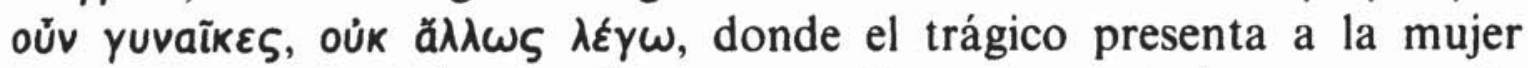
como un ser casquivano. Con estas mismas connotaciones se puede añadir asimismo el empleo por parte de Eurípides del verbo $\mu \omega p a i v \omega$ en $A n d r .674$ yuvaĩxa $\mu \omega$ paívouoav ${ }^{14}$, connotaciones que en esta familia de palabras parece testimoniar sólo este autor trágico como ya recuerdan, remitiendo a otros pasajes suyos, tanto J. Denniston, «I can find no trace of this use of $\mu \tilde{\omega}$ pos outside Euripides» como P. Stevens, «for $\mu \tilde{\omega} \rho \varsigma_{\varsigma}$ and cognates used of sexual intemperance, a sense aparently confined to Eur., see Hipp. 644, 966; Ion 545; Tr. 1059; El. 1018; fr. $331 »{ }^{15}$. En definitiva nuestra interpretación saca a la luz un nuevo y su-

" G. Mastromarco, The Public of Herondas, Amsterdam 1984, p. 78, recuerda que «the Mimiambi were in fact designed for the Alexandrian cultural élite, the only tipe of public that could appreciate the laborious search for innovations, for originality on the part of poets who intended to "walk along roads never frequented by carts"". Véanse asimismo sus observaciones en p. 74.

12 Tanto el verbo kiveiv (con la $u$. l. Bıveiv) como $\sigma \kappa \varepsilon \lambda \varepsilon a$ son frecuentes en pasa-

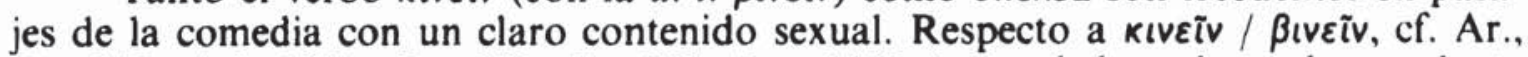
Ra. 148, Ach. 1052, Pax 341, Eq. 364, Lys. 1166 (y recuérdese el nombre parlante Kıno(as), Men., fr. 60 , Eup., fr. 247,3 , etc. Por lo que se refiere a $\sigma \kappa \varepsilon \lambda \varepsilon a$, cf. Ar., Ec. 265 y Pax 889 . Véase sobre todo ello, J. Henderson, The Maculate Muse. Obscene Language in Attic Comedy, New Haven-Londres 1975, pp. 151-152 y 173.

${ }_{13}$ Cf. J. L. Calvo Martínez, Euripides. Tragedias, vol. II, Madrid 1985 (reimpr.), p. 291, n. 6 .

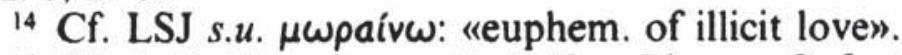

is Cf. J. D. Denniston, Euripides. Electra, Oxford 1987 (reimp.), p. 179, y P. T. Stevens, Euripides. Andromache, Oxford 1984 (reimp.), p. 177. 
til efecto humorístico no apreciado hasta ahora en el pasaje de Hds. para el cual se puede postular asimismo que el poeta, en su gusto por las rarezas, está siguiendo un uso infrecuente de la palabra $\mu \tilde{\omega} \rho{ }^{\circ} \varsigma$ que, hasta ahora, se consideraba exclusivo de Eurípides. Hds. nos muestra con todo ello su condición de genuino poeta doctus.

Luis Alfonso Llera Fueyo 